\title{
Niñez indígena y desnutrición Análisis antropológico comparativo de la implementación de programas alimentarios en Colombia y Argentina
}

María Pía Leavy, ${ }^{1}$ Andrea Paola Szulc ${ }^{1,2}$ Ingrid Anzelin ${ }^{*}$

\author{
1 Universidad de Buenos Aires, Buenos Aires, Argentina. \\ Correo electrónico: pialeavy@gmail.com \\ 2 Consejo Nacional de Investigaciones Científicas y Técnicas, Argentina \\ Correo electrónico: andrea.szulc@gmail.com \\ 3 Universidad de La Sabana, Chía, Colombia. \\ Correo electrónico: ingrid.anzelin@unisabana.edu.co
}

Recibido

octubre de 2017

Aceptado

mayo de 2018

\section{Resumen}

El presente artículo presenta un análisis antropológico sobre programas alimentarios dirigidos a niños y niñas indígenas de zonas rurales de la provincia de Salta (Argentina) y del departamento de Guainía (Colombia). Desde un enfoque etnográfico, se utilizan técnicas cualitativas para observar la implementación del Plan Nacional de Seguridad Alimentaria, en el caso argentino, y el programa de Hogares Comunitarios de Bienestar, en el caso colombiano. El análisis antropológico permite observar que el reconocimiento especial de derechos a niños y niñas indígenas se traduce en políticas focalizadas hacia los cuerpos infantiles, que omiten los procesos históricos, sociales y políticos que llevaron a que las poblaciones indígenas vivan en condiciones de inseguridad alimentaria. Planteamos entonces que el cuidado infantil configura un campo de disputas entre agentes estatales y adultos cuidadores donde la alteridad indígena es comprendida como la causa de la emergencia de los déficits nutricionales.

\section{Indigenous Childhood and Malnutrition: anthropological compara- tive analysis of the implementation of food programs in Colombia and Argentina}

\author{
Abstract \\ This article presents an anthropological analysis of food assistance programs for indige- \\ nous children from rural areas in the province of Salta, Argentina, and the department \\ * María Pía Leavy: Doctora y profesora en Ciencias Antropológicas por la Universidad de Buenos Aires. Docente en \\ la Maestría de Problemáticas Infanto-Juveniles de la Facultad de Derecho (UBA). \\ Andrea Paola Szulc: Investigadora adjunta del CONICET en el Instituto de Ciencias Antropológicas, FFyL, UBA. \\ Doctora y licenciada en Ciencias Antropológicas (UBA). \\ Ingrid Anzelin: Licenciada en Psicología y Profesora en la Facultad de Educación de la Universidad de La Sabana.
}

\section{Palabras clave}

Infancia; Etnografía; Programas Alimentarios; Pueblos Indígenas; Cuidado

\section{Key words}

Childhood; Ethnography; Food assistance programs; Indigenous peoples; Childcare 
of Guainía, Colombia. An ethnographic approach is used to assess the implementation of the National Food Security Plan in Argentina and the Community Wellbeing Homes in Colombia. Anthropological analysis brings into focus how the recognition of special rights of indigenous children translates into policies focused on children's bodies that overlook the historic, social and political processes that led indigenous populations to suffer from food insecurity. Thus, we suggest that childcare configures a contested field between state officials and adult caregivers, in which indigenous otherness is understood as the main cause of malnutrition.

\section{Infância Indígena e Desnutrição. Análise antropológica compara- tiva da implementação de programas de alimentos na Colômbia e na Argentina}

\section{Resumo}

Palavras-chave

Infância; Etnografia; Programas Alimentares; Povos Indígenas; Cuidado
Este artigo apresenta uma análise antropológica de programas alimentares orientados a crianças indígenas de zonas rurais da província de Salta (Argentina) e da província de Guainía (Colombia). A partir de um enfoque etnográfico, utilizam-se técnicas qualitativas para observar a implementação do Plano Nacional de Segurança Alimentar no caso argentino e do programa Lares Comunitários de Bem Estar no caso colombiano. A análise antropológica permite observar que o reconhecimento especial de direitos a crianças indígenas traduz-se em políticas focadas nos corpos infantis e que omitem os processos históricos, sociais e políticos que levaram as populações indígenas a viverem em condições de insegurança alimentar. Propomos então que o cuidado infantil configura um campo de disputas entre agentes estatais e adultos cuidadores no qual a alteridade indígena é compreendida como a causa dos déficits nutricionais.

\section{Introducción}

Desde inicios del siglo XXI, se han implementado diversos programas que buscan garantizar la seguridad alimentaria en el contexto latinoamericano y específicamente hacia pueblos indígenas (Comisión Económica para América Latina y el Caribe [CEPAL], 2007; Organización de las Naciones Unidas para la Alimentación y la Agricultura [FAO], 2015). Si bien el concepto de "seguridad alimentaria" se asocia al derecho universal de todas las personas a acceder a una alimentación adecuada en términos culturales y biológicos (Calvo y Aguirre, 2005), en la práctica y con frecuencia, el accionar estatal se focaliza exclusivamente en la población infantil (Osorio Figueroa y Bahía Coelho, 2017) y en ocasiones aún más, en la población infantil indígena menor de seis años de edad (Leavy, 2015). El presente artículo presenta entonces un análisis antropológico sobre cómo se han focalizado ciertos programas alimentarios en niños y niñas indígenas de zonas rurales de la provincia de Salta (Argentina) y del departamento de Guainía (Colombia) y las tensiones que ello suscita en su implementación.

Nuestra perspectiva antropológica nos llevará a analizar la noción de infancia que subyace a la puesta en práctica de dichas políticas públicas y que se articula con determinadas nociones sobre el cuidado, la salud y la alimentación. Utilizaremos entonces un enfoque etnográfico, que somete los conceptos supuestamente universales y naturales - como el de niñez o infancia - a la diversidad de la experiencia humana (Guber, 2001), 
al reintroducir el sentido que ella tiene para los sujetos. Asimismo, dicho enfoque nos permite relevar las prácticas y representaciones que en cada contexto constituyen la niñez, lo cual incluye no solo el accionar institucional y de los adultos, sino asimismo los modos en que los niños y niñas experimentan las realidades en que viven (Szulc, 2006).

\section{Consideraciones metodológicas}

En el caso argentino, el abordaje etnográfico fue realizado por Pía Leavy en el marco de su investigación doctoral, en el que analiza el cuidado y la nutrición infantil en poblaciones indígenas y criollas en contextos rurales del departamento de Orán, Salta, en el período 2010-2015. En el caso colombiano, el material etnográfico fue producido por Ingrid Anzelin en 2006, en el marco del programa "Colombia por la primera Infancia”, que tenía como objetivo conocer experiencias catalogadas como exitosas de los programas del Instituto Colombiano de Bienestar Familiar (ICBF) en diversos departamentos del país. Además de las técnicas de observación participante y entrevistas individuales y grupales, hemos utilizado técnicas de documentación (Valles, 2000) para analizar los documentos teóricos de ambas políticas públicas, los portales web de los organismos estatales responsables y diversos informes técnicos.

Más allá de las diferencias registradas entre los gobiernos argentinos y colombianos en el período aquí analizado, las intervenciones alimentarias estatales evidencian marcadas similitudes en su implementación, focalizadas en poblaciones vulnerables. Asimismo, en ambos casos, las poblaciones indígenas han sufrido históricamente procesos de expulsión y han sido desplazadas de sus tierras originarias. El presente ejercicio comparativo - cuyo fin es contribuir a los estudios sobre la desnutrición en el contexto latinoamericano, que tienden a encarar la cuestión solo de modo cuantitativo - tuvo su origen en las semejanzas que presentan los procesos generadores de la inseguridad alimentaria y la aplicación de políticas ultrafocalizadas propias de la gubernamentalidad neoliberal.

\section{El caso argentino: Barrio "La Fortaleza"1}

\section{1) Descripción de las acciones estatales analizadas}

Las políticas sociales implementadas en Argentina en materia de alimentación constituyen un conjunto heterogéneo de programas y acciones, que desde el año 2003 se nuclean bajo el Plan Nacional de Seguridad Alimentaria (PNSA-Ley No 25.724). Dicho plan fue reglamentado en un contexto de fuertes presiones sociales, luego de la declaración de la emergencia alimentaria nacional, y apuntó a constituirse como "la" política de Estado en materia alimentaria, mediante la unificación de los programas hasta entonces vigentes. ${ }^{2}$ El objetivo central del PNSA se planteó como asegurar el acceso a una alimentación adecuada y suficiente, coordinando desde el Estado las acciones integrales e intersectoriales que facilitasen el mejoramiento de la situación alimentaria y nutricional de la población (Decreto 1018/03, art. 1º).

Este programa tuvo la particularidad de ser uno de los primeros en Argentina en ubicar a los niños como "titulares de derechos", junto con sus familias (Ministerio de Desarrollo de la Nación, 2015), y marcó un antecedente a la sanción de la Ley № 26.061 (2005), de Protección Integral de los Derechos de las Niñas, Niños y Adolescentes, que inauguró el amplio conjunto de políticas públicas que buscaron "garantizar el interés superior del niño" (Llobet, 2011). Así pues, el PNSA ubica como titulares de derechos tanto a niños como a sus familias, junto con otras poblaciones vulnerables. ${ }^{3}$ En este sentido, encontramos el subprograma Focalizado Aborigen dentro del PNSA en el
1. A fin de preservar el anonimato de nuestros/as interlocutores/ as, los nombres de personas y lugares han sido modificados.

2. Aunque fue elaborada por expertos en la temática, la ley 25.724 se denominó “El Hambre más Urgente" gracias a gestiones de ONG como Poder Ciudadano, medios de comunicación como La Nación, el canal América TV, así como por algunas empresas, entre ellas, Metrogas, Metrovías y Fundación Andreani, que consiguieron el número de firmas equivalente al 1,5 del padrón electoral y luego la reglamentación en abril de 2003 mediante el Decreto № 1018/03 (Patricia Aguirre, 2014, com. pers.).

3. Las titulares de derecho de esta acción son aquellas familias con niños menores de 14 años, mujeres embarazadas, personas con discapacidad y adultos en condiciones socialmente desfavorables y de vulnerabilidad nutricional (Ministerio de Desarrollo Social, 2009). 
4. El Programa de Atención Primaria de la Salud se desarrolla en Argentina desde principios de la década de 1980 y comprende la asistencia sanitaria esencial y accesible a toda la población sin cobertura privada, que constituye el primer contacto entre la comunidad y los servicios de salud. Las principales actividades desarrolladas incluyen la educación para la salud, la prevención y control de enfermedades y el suministro de medicamentos e inmunizaciones. Para profundizar, véase Torres Aliaga y Torres Secchi (2010), Maceira (2010).

5. Para el año 2015, "la media nacional de diferencia entre los ingresos de los más ricos y los más pobres es de un cociente de 20 , mientras que el de la provincia de Salta es de 30, y es el más elevado del país" (El Tribuno Salta, 21 de abril de 2015). Por otro lado, la Encuesta Permanente de Hogares (EPH-INDEC) del cuarto trimestre del 2014 reveló que, en Salta, el $10 \%$ de la población más rica se lleva el $35.3 \%$ de los ingresos brutos, mientras que en el otro extremo, el $10 \%$ más pobre, se lleva solo el 1,2\% (INDEC, 2014).

6. Salta se caracteriza por ser la "provincia récord en desmonte", ya que entre 2008 y 2013 se desmontaron 358.723 hectáreas, de las cuales 112.000 estaban en zonas que debían protegerse según el ordenamiento territorial de la Ley de Bosques (Ley Nacional № 26.331) (Aranda, 2014).

7. Los factores de riesgo son: "Grupo integrado por menores de 6 años"; "Desempleo y/o ingreso económico inestable (trabajo irregular, jornalero)"; “Ausencia de Padre o Madre (Padre o Madre soltero/a- Viudo/a)"; "Carencia de Obra Social”; "Enfermedades crónicas en la familia"; "Enfermedades Sociales"; "Niños menores de 6 años con déficit nutricional"; "Embarazada"; "Tratamiento Inadecuado de Agua, Residuos";

"Analfabetismo de la persona a

cargo de los menores"; "Morta-

lidad Infantil/ Materna"; "Recién

nacido/Puérpera" (Formulario

N.1). Para problematizaciones sobre esta dinámica de gobierno, ver Lorenzetti (2015). contexto de Salta, que tuvo por objetivo el suministro de módulos alimentarios a familias indígenas de los departamentos de San Martín, Santa Victoria y de Orán (Aulicino, 2012), donde se concentran las poblaciones más vulnerables.

El PNSA articulaba entonces acciones directas e indirectas. Entre las primeras encontramos líneas de financiación a través de transferencias bancarias y una multiplicidad de acciones en terreno que implicaban la articulación de los tres niveles gubernamentales (nación-provincia- municipio) para desarrollar las prestaciones de asistencia alimentaria y nutricional directa (entrega de módulos alimentarios o transferencias monetarias); asistencia a comedores sociales e infantiles; autoproducción de alimentos; mejoramiento de los servicios alimentarios de comedores escolares y cuidado de la embarazada y el niño sano (Aulicino, 2012). Entre las acciones indirectas, destacamos la regulación del valor del dólar (aspecto fundamental en el funcionamiento de la industria alimentaria) y la quita de impuesto al valor agregado en los alimentos de la canasta básica.

\section{2) Contextualización del área de estudio}

En la provincia de Salta, ubicada en el noroeste del territorio argentino, el PNSA ha sido implementado por técnicos del Ministerio de Desarrollo Social de la Provincia, en articulación con los agentes sanitarios del Programa de Atención Primaria de la Salud $^{4}$ (PROAPS). Esta provincia se destaca por la amplia diversidad de pueblos indígenas que viven en ella - ava-guaraní, tapiete, wichí, toba-qom, chorote, chulupí-, así como también por presentar altos niveles de desigualdad en la distribución de los ingresos. ${ }^{5}$ Esta desigualdad se expresa en la concentración de las tierras productivas en unos pocos conglomerados industriales-agrícolas y en la endeble posición de los pueblos indígenas ante al avance de frentes extractivos madereros, agrícolas y de hidrocarburos. ${ }^{6}$ De este modo, las comunidades indígenas no integran una homogeneidad étnica, sino más bien un mosaico de identidades de diferentes grupos humanos que comparten un espacio por cuestiones sociales e históricas (Cravino, 2008). Los actuales vecinos de Fortaleza - el barrio donde se realizó el trabajo de campo-vivían previamente sobre terrenos por donde pasaba el gasoducto Norandino. Debido a la peligrosidad de la zona, diversas organizaciones sociales e indígenas se movilizaron exigiendo el derecho a una vivienda digna, y lograron que en 2006 los gobiernos nacional y provincial se comprometieran a construir un barrio de viviendas. El barrio construido, de 400 casas, se localizó en medio de un campo donde se plantaba soja, de modo que los vecinos estaban expuestos al uso de agroquímicos implicado en dicha actividad económica. Allí se construyó también un Centro de Atención Primaria de la Salud (CAPS) - donde todas las mañanas atendía un enfermero y desde donde los agentes del PROAPS organizaban su atención sanitaria (Leavy, 2014) -, pero aún está pendiente la construcción de una escuela primaria.

\section{3) La focalización desde la mirada etnográfica}

Para los agentes del PROAPS, Fortaleza se dividía en tres "sectores de salud", en cada uno de los cuales vivían aproximadamente cien familias. Estos sectores eran responsabilidad de tres agentes sanitarios, que nucleaban sus actividades en el CAPS, donde guardaban los registros y formularios con los cuales clasificaban a la población paciente, junto con las inmunizaciones y medicaciones necesarias para su atención. Felisa, agente sanitaria ava-guaraní, tenía a su cargo un sector integrado por 118 familias, que eran clasificadas de acuerdo con los "factores de riesgo" elaborados por el PROAPS para definir dónde debía focalizar las visitas domiciliarias cada agente sanitario. ${ }^{7}$

Así, la presencia de "niños recién nacidos y madres puérperas" constituye hasta el presente un factor de focalización de las acciones del PROAPS en un determinado 
grupo familiar. La atención sanitaria hasta los seis años se concentraba en la aplicación de las inmunizaciones que corresponden al calendario obligatorio y la realización de controles nutricionales. Estos implicaban la medición de talla y peso una vez al mes durante el primer año de vida. ${ }^{8}$ Los niños que presentaban diagnósticos de desnutrición crónica y/o grave debían ser controlados cada quince días.

Las medidas de talla y peso eran registradas por los agentes de salud en sus formularios y en la "curva de crecimiento" consignada en los carnets de salud de los niños y niñas pacientes. Según esta curva, la desnutrición se medía en leve, moderada y grave (Niveles $1,2,3$ ). El grado 1 (leve) se utilizaba para clasificar a los niños que no habían alcanzado un peso acorde con su talla. El grado 2 (moderada) indicaba que un niño menor de un año tenía peso bajo para su edad y se utilizaba también para señalar una relación baja de peso/talla. La desnutrición grave, o grado 3, indicaba que un niño menor de un año presentaba un déficit del $40 \%$ o más del peso ideal para su edad.

Cabe destacar que en el año 2012, desde el Ministerio de Salud de la provincia de Salta se dispuso "no hablar más de desnutrición, sino de déficits nutricionales y casos de sobrepeso" (EA, 22/3/2013). Asimismo, las señaladas clasificaciones por medio de los grados de desnutrición fueron reemplazadas por "Bajo Peso" (grado 1 y 2) y "Muy Bajo Peso" (grado 3). Si bien excede a los objetivos del presente trabajo profundizar en las implicancias de dicho cambio de denominación, resulta relevante señalar que estas clasificaciones son determinantes en la cantidad de módulos alimentarios que se entregan a cada familia. ${ }^{9}$

En una oportunidad, acompañando a Felisa en su "ronda sanitaria"10 por la Fortaleza, la antropóloga Pía Leavy visitó una casa donde vivían “dos grado tres”, como les decía la agente sanitaria a los niños "en riesgo". Se trataba de Yonatan, de 14 meses, y María, de 8 meses. Ambos niños eran primos, integrantes de una familia wichí donde vivían cuatro generaciones: abuela, madre, tres hijas y cinco nietos, entre los que se encontraban los niños “en riesgo". Mientras Felisa aplaudía para avisar que quería entrar a la casa y estaba llegando a iniciar la visita médica, le decía a Leavy al oído:

Acá vas a ver, es como que los más guapos y ordenados son los kollas y los criollos, luego venimos nosotros, los guaraníes, y los wichís a lo último, no quieren hacer nada, ni limpiar su casa y tienen a los niños como animalitos. (Felisa, Registro de campo, septiembre de 2012)

Con esta introducción al organigrama social de alteridades salteñas, ingresaron al comedor de la vivienda, donde había tres sillas, una mesa y dos reposeras, que rodeaban un televisor prendido. Al fondo de la sala estaba la cocina y la pileta, pero sin conexión a gas, por lo tanto, tenía un uso más de alacena que de horno. En el patio exterior, una mujer (la bisabuela de los pacientes) estaba haciendo un fuego y cortando verduras para hacer un guiso con fideos secos. Al costado del televisor estaba el "bolsón de comida", abierto, que exponía que ya habían sido utilizados varios paquetes. Había tres niños en el piso, gateando y jugando con unas cajas de cartón y algunos paquetes del bolsón.

Felisa tomó una de las sillas mientras saludaba a "las mamis"; apoyó su bolso en la mesa y comenzó a sacar las carpetas donde tenía los formularios de sus pacientes. Una vez desplegados estos sobre la mesa, buscó un marco donde poder colgar el gancho de la balanza. Mientras probaba si la balanza estaba colgada de forma segura, le pidió a una de las madres - la que estaba barriendo- que trajera los carnets de salud de Jonathan y de María. Claudia, la mamá de Yonatan, trajo el carnet envuelto en una bolsa de plástico y ayudó a Rosa a sujetar al niño al centímetro de madera apoyado en el piso. Las dos mujeres agachadas sujetaron al niño - que gemía, como llorando- contra el piso.
8. La medida del peso, la talla y el coeficiente de índice corporal resultan los indicadores poblacionales más extendidos, recomendados desde organismos internacionales, puesto que son fáciles de determinar, de muy bajo costo y de alta sensibilidad (Ministerio de Salud, 2007). Las guías utilizadas se denominan NHS (por sus siglas en inglés, National Health Service) y permiten medir los valores antropométricos en referencia con una población muestral que presenta una distribución normal. El límite o punto de corte para distinguir lo normal y lo patológico se define como una distancia determinada con respecto al valor promedio. La distancia puede estar expresada bajo la forma de desviaciones estándar, de percentiles o de porcentaje de adecuación a la mediana (O’Donnell y Carmuega, 1998; Herkovits, 2008).

9. Al momento de realizar el trabajo etnográfico, cada módulo alimentario incluía $2 \mathrm{~kg}$ de sémola, 2 kg de azúcar, 1 kg de arroz, 2 litros de aceite de maíz, 4 kg de fideos secos, $500 \mathrm{~g}$ de membrillo, 2 latas de duraznos en almíbar y 2 $\mathrm{kg}$ de leche fortificada. En el año 2013, todos los niños menores de dos años recibían una vez por mes un kilo de leche en polvo. Aquellos niños con diagnóstico grado 2 recibían dos kilos por mes, mientras que aquellos que presentaban grado 3 , recibían $3 \mathrm{~kg}$ por mes.

10. Es el nombre que recibe el trabajo de visitar las viviendas cotidianamente. 
Felisa anunció en voz alta el veredicto: “76 centímetros”. Fue hasta su carpeta y anotó la talla, al igual que en el carnet de salud del niño.

Está bajo, mami, bien bajo, esto es muy grave, mami. Él no ha crecido desde la última vez, ahora tenemos que ver el peso, pero no sé mami si habrá buenas noticias y yo miro todo esto (mira el paquete abierto) y pienso, ¿por qué hacen esto mami?, ¿por qué comen lo que es del niñito?, ¿por qué hacen eso?, si les damos comida es para el niño, no para toda la familia, ¿por qué están todos comiendo lo que es de él? (Felisa, Registro de campo, septiembre de 2012)

Felisa preguntaba señalando el plástico roto del módulo alimentario. La madre de Yonatan miraba en silencio, las otras mujeres alrededor (la bisabuela en el fuego, en el patio y la otra barriendo) continuaban sus actividades. “ ¿Ves lo que pasa?” dijo Felisa a la observadora como ejemplificándole en vivo y en directo los conflictos de su trabajo. Leavy, que estaba sentada en el piso jugando con uno de los nenes, se quedó callada, al igual que las madres de los niños desnutridos; la situación era extremamente incómoda.

Cuando salieron de la casa, Felisa expresó, con un tono de preocupación, tanto por el niño como por la utilidad de su trabajo:

A veces siento que los bolsones sólo trajeron más desnutridos, esos changuitos ${ }^{11}$ ahí comiendo en el piso como animalitos, todo sucio, mal cuidado, siento que no sirve lo que hacemos, ellos (población paciente) no van a dejar de compartir la comida y entonces el chiquito nunca va a crecer bien, no sirve nada de lo que hago entonces, ya no sé para qué lo hago. (Felisa, Registro de campo, septiembre de 2012)

\section{El caso colombiano: Puerto Inírida}

\section{1) Descripción de las acciones estatales analizadas}

Las políticas alimentarias analizadas para el caso colombiano se enmarcan en el programa de Hogares Comunitarios de Bienestar (HBC), que surgió a finales del año 1986, con la aprobación del Consejo Nacional de Política Económica y Social (CONPES), como estrategia de "desarrollo humano" integral dirigida a atender a los niños meno-

12. Según Castillo Matamoros (2009), el programa pretendió acompañar el proceso de socialización y mejorar tanto la nutrición como las condiciones de vida de estos niños mediante acciones realizadas por "las madres comunitarias". El Instituto Colombiano de Bienestar de la Familiar (ICBF) se financia con el $1 \%$ adicional de los recursos generados por las nóminas mensuales de las empresas públicas y privadas (Castillo Matamoros, 2009). res de 7 años pertenecientes a las poblaciones más pobres del país. ${ }^{12}$ Los HCB se caracterizan por brindar a niños y niñas menores de seis años — según parámetros “de necesidad" (pobreza, estratificación económica, necesidades de sus familias, deficiencias nutricionales de los niños, etc.) - cuidado y atención, con un componente importante de asistencia nutricional de acuerdo con la cual se determinan raciones de comida por cada niño con el fin de lograr recuperación nutricional en casos de desnutrición crónica o aguda y evitar que eso ocurra a niños y niñas en "condiciones de vulnerabilidad"(Instituto Colombiano de Bienestar Familiar, 2014). Dentro de esta estructura, se asigna a una madre de la comunidad para que cuide entre doce y catorce niños en su casa por un número determinado de horas, en las que les brinda cuidados y la alimentación (Instituto Colombiano de Bienestar Familiar, 2014).

En la actualidad, el funcionamiento de los HCB se enmarca en el programa "De Cero a Siempre", que reúne políticas, programas, proyectos, acciones y servicios dirigidos a la primera infancia, con el fin de prestar una verdadera Atención Integral que haga efectivo el ejercicio de los derechos de los niños y las niñas entre cero y cinco años de edad (Instituto Colombiano de Bienestar Familiar, 2014).

Desde los documentos iniciales, el programa sostiene que "la inversión en la primera infancia es el aporte más rentable a largo plazo para un país", y que tanto "la familia, 
como la sociedad y el Estado están en la obligación de garantizar la protección, la salud, la nutrición y la educación inicial desde el momento de la gestación hasta los cinco años" (Instituto Colombiano de Bienestar Familiar, 2014). El desafío del programa consiste en coordinar las acciones ya existentes - como aquellas dependientes del Instituto Colombiano de Bienestar Familiar (ICBF), tal como los $\mathrm{HCB}$ - promoviendo la universalización de la atención integral a niños y niñas. Cabe destacar que, si bien se propone la universalización, desde los lineamientos teóricos se prioriza la atención sobre la población "en Pobreza Extrema". ${ }^{13}$ La selección de la población destinataria se realiza mediante el Sistema de Identificación de potenciales Beneficiarios de Programas Sociales (SISBEN).

\section{2) Contextualización del área de estudio}

De acuerdo con el relevamiento realizado por el Departamento de Administración Nacional de Estadísticas (DANE) en 2005, aproximadamente el 64,9\% (11.595 personas) de la población de Guainía eran indígenas. "Así, Guainía es una de las regiones más multiculturales y multilingües de Colombia" (Daza Ramos, 2015, p. 5), a la vez que una de las más vulnerables y escasamente desarrolladas, con altas tasas de pobreza y analfabetismo (Daza Ramos, 2015). Según un reciente informe de la Food and Agriculture Administration (FAO) (2015), en Colombia, "la cifra promedio de desnutrición crónica en niños y niñas menores de cinco años, esconde grandes variaciones. Existen departamentos que presentan un retraso en crecimiento superior al $20 \%$, que es considerado a nivel internacional como una prevalencia mediana” (FAO, 2015, p. 36).

La población indígena entre la que se realizó la observación pertenece al pueblo puinave, que habita en un asentamiento a las orillas del río Inírida, bajo río Guaviare, ubicado en la cuenca del río Orinoco. El asentamiento de población puinave en esta localidad está vinculado a procesos de desterritorialización, generados por el conflicto armado entre la guerrilla y el gobierno colombiano y la producción de coca para el narcotráfico. Referentes locales también vienen señalando el impacto de la actividad minera en las condiciones de vida de esta población:

La zona rural es inmensa, ríos y selvas es lo que tiene Guainía, y comunidades indígenas. Pero los ríos son pobres en pesca, la seguridad alimentaria es bien deficiente. Los ríos son oscuros y con bajos nutrientes, y lo peor es que hay minería con dragas que agrava la situación. (El Tiempo, 14/3/2016)

En ese contexto, fuentes periodísticas señalan que el ICBF — con responsabilidad en estos temas de nutrición infantil- solo tiene tres madres sustitutas para atender a los niños desnutridos y cuatro hogares comunitarios a los que asisten 1000 niños. Es de destacar que algunos funcionarios señalan a la población indígena como responsable, en parte, de esta situación:

Existió bonanza cocalera y los indígenas abandonaron la producción de especies alimenticias. En Guaviare había bastante producción de cacao y ya no. Incluso había una especie de batata (llamada papa aérea), había ñame en esta región, pero esos productos han desaparecido porque los indígenas no los volvieron a cultivar. Eso sólo trae pobreza y por ende desnutrición. Es una tarea y un reto la producción de alimentos en estos suelos. (El Tiempo, 14/3/2016)

\section{3) La focalización desde la mirada etnográfica}

Con el objetivo de garantizar los derechos de la infancia, los HCB tienen por objetivo ofrecer una "atención diferencial en primera infancia a comunidades étnicas", que implica el reconocimiento de sus particularidades y formas de vida (El Tiempo, 14/3/2016).
13. Según se plantea en el sitio web oficial, "sólo el $24 \%$ los niños y niñas menores de cinco años recibe atención integral y De Cero a Siempre buscará hacer efectivos los derechos a la atención integral de 1.500 .000 niños y niñas en situación de vulnerabilidad, enfrentándose así a un gran reto Nacional que exige una coordinación interinstitucional para alcanzar una cobertura del 100\% de la población entre cero y cinco años de edad". En: http:// www.deceroasiempre.gov.co/ Prensa/CDocumentacionDocs/ Fundamientos-politicos-tecnicosgestion-de-cero-a-siempre.pdf. 
Tal es el caso en Puerto Inírida, según ha registrado Ingrid Anzelin, donde la atención a niños y niñas no se realizaba en la casa de una madre comunitaria, sino en la maloka de la comunidad puinave Cunare, donde se convocaba cotidianamente a niños y niñas a que realicen sus comidas.

Si bien la experiencia "intercultural" del ICFB en la comunidad Cunare era catalogada por los funcionarios como exitosa, los mismos agentes del ICFB de Puerto Inírida dudaban de la eficacia del "servicio de atención" en esa localidad:

El Instituto nos da cantidades específicas de alimento por el número de niños que van a este HCB, pero nos hemos visto inquietados porque una vez les damos el alimento a los niños estos se 'escapan' de la maloka y van corriendo a sus casas y reparten con los miembros de su familia lo que les damos [...]. A mí me contrata el Estado a través del ICBF para garantizar que los pocos recursos que llegan a este Departamento (Guainía, en donde hay baja presencia del Estado) estén donde se dicen que están, y no puedo seguir justificando las raciones de comida sabiendo que no están llegando adonde tienen que llegar y es a los niños. Las tablas de talla y peso están igual siguen igual de desnutridos a cuando llegaron al programa. (Agente estatal del ICBF, comunidad puinave, septiembre 2006)

Con estas palabras, la funcionaria manifestaba una profunda preocupación porque los niños atendidos repartían su ración de comida entre seis o siete personas, o más, de tal forma que nadie se alimentaba adecuadamente. Así pues, el niño continuaba con los mismos índices de desnutrición. La funcionaria contaba que, ante este panorama, habían intentado cambiar la metodología de los HCB, pero que el ICFB era rígido con sus lineamientos y la instalación del HCB en la maloka ya era demasiado.

En relación con esta tensión entre la propuesta del "servicio de atención" y cómo los destinatarios/usuarios hacían uso de dicho servicio, una madre expresó:

El tema de la comida es muy importante, dado que se le enseña a los niños que lo que es de uno es de todos, así pues no está bien visto que 'coman solos' y que mientras ellos comen el resto de su familia tiene hambre. (Marisa, marzo de 2006)

Asimismo, la madre comentaba que varias veces habían tratado de ponerse en las salidas de la maloka (que no tiene puertas) para evitar que los niños y niños salieran. Como respuesta a esta actitud, los niños se ponían la comida en los bolsillos y así la llevaban hasta sus casas. Al respecto, la madre agregaba:

No los podemos obligar a que coman, o que cuando coman sean mal vistos por su familia o ellos se sientan mal por tener más "beneficios" que los demás miembros de su familia que están en las mismas condiciones de ellos. (Marisa, marzo de 2006)

\section{Análisis de los casos}

\section{1) Derechos especiales y gubernamentalidad neoliberal}

La seguridad alimentaria ha sido reconocida como parte de los derechos especiales de los niños y de los pueblos indígenas (ONU, 2016). Este "reconocimiento especial" implica garantizar un "plus" de derechos a estas poblaciones que presentan mayores vulneraciones por motivos históricos, políticos y sociales. Ahora bien, en ambos casos, podemos observar que el reconocimiento especial que promueven los derechos se traduce en la implementación de políticas sociales que se focalizan sobre los más vulnerables. En el caso argentino, la focalización se realiza a través de las mediciones 
de factores de riesgo del PROAPS, mientras que en el caso colombiano, a través de las mediciones del SISBEN.

Resulta relevante señalar que, en el caso argentino, la implementación de las acciones directas del PNSA se dio en paralelo a acciones indirectas, como ya mencionamos, y otras como la Asignación Universal por Hijo, desde la cual se modificó el régimen de asignaciones familiares, ampliándolas a hijos de trabajadores no registrados en la seguridad social, desocupados y empleados/as domésticas/os (Andiñach, 2014). Sin embargo, el material etnográfico presentado permite observar que las acciones en territorio se organizan a través de una "foco-política” (Álvarez Leguizamón, 2011), que define intervenciones a través de sumatorias de vulnerabilidades, ya sea por cohortes de edad o por niveles de pobreza. Según Álvarez Leguizamón, la foco-política dirigida al gobierno de la pobreza creciente constituye una nueva práctica del derecho político supranacional y un nuevo humanitarismo, bajo una fuerte influencia de los organismos supranacionales "que dicen promover el desarrollo de la vida pero lo hacen a niveles mínimos básicos cercanos a la desnutrición” (Álvarez Leguizamón, 2011, p. 265). Así pues, señalamos la particular noción de sujeto que subyace a estas prácticas focalizadas, propias de la gubernamentalidad neoliberal (Rose, O Malley y Valverde, 2006), que suponen un destinatario empresario de sí mismo, que debe autogestionar su salud y su alimentación. La focalización traslada al "vulnerable" la resolución individual, familiar o vecinal de su problema y no contempla una regulación estatal en la relación capital/ trabajo (Álvarez Leguizamón, 2005).

En los casos de los niños indígenas aquí abordados evidenciamos cómo la responsabilidad de sus padecimientos, así como los déficits nutricionales, era atribuida por los agentes estatales en territorio al cuidado inadecuado de sus madres u otros familiares a cargo. En este sentido, Álvarez Leguizamón (2011) sostiene que la práctica focalizadora de las políticas gubernamentales “inferioriza las capacidades culturales (estilos de vida) de sus destinatarios (pobres) y produce una estigmatización de los mismos, al ser recortados de un conjunto, seleccionados y distinguidos por su incapacidad de sobrevivencia autónoma" (Álvarez Leguizamón, 2011, p. 262, destacado en el original). Desde una perspectiva antropológica, sin embargo, debemos advertir que los obstáculos de la población destinataria "para sobrevivir autónomamente" no derivan de la supuesta "incapacidad" para cuidar a sus hijos, ${ }^{14}$ sino más bien de los procesos señalados anteriormente, vinculados al despojo territorial y al deterioro ambiental.

De este modo, el "plus" de derechos del reconocimiento especial, que supuso el proceso de institucionalización por medio de políticas públicas, se traduce en una intervención estatal que busca garantizar los derechos básicos, como salud y acceso a agua potable. Así, iniciativas enunciadas como "políticas de reconocimiento" acaban muchas veces reduciéndose a políticas sociales (Lorenzetti, 2007), como hemos observado también en la perspectiva con que organismos internacionales como UNICEF abordan los derechos de los niños indígenas (Szulc, Colangelo, Shabel, Leavy, Enriz, Hernández, 2016).

\section{2) El niño como cuerpo individual}

La noción de salud que promueven las políticas públicas analizadas está estrechamente ligada al control nutricional de los cuerpos infantiles, que son los que determinan el estado (in)adecuado del crecimiento y desarrollo. Desde la perspectiva antropológica, diversos estudios han afirmado que el "crecimiento" y el "desarrollo" constituyen las dos metáforas con las cuales las sociedades han abordado la infancia, como símbolo de futuro (Jenks, 1996; Colangelo, 2008). Coincidimos con el saber biomédico en que, si un humano no se alimenta adecuadamente en sus primeros años de vida, sufrirá alteraciones en su crecimiento y desarrollo que le dificultarán su aprendizaje y su vida futura.
14. En su labor como perito antropológico en la Comisión de Derechos Humanos de la Cámara de Diputados del Congreso brasileño para presentar un argumento que clarificase a los parlamentarios sobre el supuesto "infanticidio indígena”, Segato (2013) sostiene que no hay modo más eficaz de subestimar a una minoría étnica, sea cual fuera su origen, que aludir a su irresponsabilidad para cuidar a la descendencia, que construye "la supuesta necesidad de proteger al indio de su incapacidad cultural para cuidar la vida" (Segato, 2013, p. 118). 
15. Ver https://youtu. be/7JZEHly1pfl recuperado de http://www.deceroasiempre.gov. co/Paginas/deCeroaSiempre.aspx.
No obstante, advertimos que la focalización de las intervenciones en los cuerpos infantiles implica una mirada descontextualizada de sus vidas.

En ambos casos, los niños viven en contextos de inseguridad alimentaria. Por lo tanto, ellos y sus familiares sufren dificultades para acceder a una alimentación adecuada. Resulta curioso entonces que, aun cuando en los diversos materiales abundan las referencias a la infancia en términos afectivos, ${ }^{15}$ no se considere el deseo de los niños de compartir la comida con sus familiares.

Como advierte Fassin (2003), estas acciones — propias de la racionalidad neoliberalconfiguran un orden moral en el cual el plano de lo biológico, representado por el cuerpo sufriente, constituye la fuente de legitimidad. Así pues, las acciones de asistencia alimentaria configuran una "política de reconocimiento del ser sufriente y del cuerpo enfermo que puede entenderse como parte de las formas que adquiere la biolegitimidad" (Fassin, 2003, p. 310), ya que son los cuerpos con diagnóstico de bajo peso los más legítimos destinatarios de las acciones de gobierno. Resulta interesante que, en ambos casos, los agentes estatales sienten frustración cuando los niños comparten comida con sus familias, cuando el hecho de compartir alimentos y la reciprocidad son cuestiones que numerosos autores vienen hace tiempo señalando como elemento clave en los modos de vida de los pueblos indígenas (Lee, 1981; Ingold, 1988), persistente a pesar de su incorporación subordinada a sociedades capitalistas periféricas (Gordillo, 2006). Al mismo tiempo, en los contenidos escolares y en el cotidiano escolar de nivel inicial y primario "el compartir" se enfatiza y transmite a todos los niños como contenido actitudinal crucial a aprender, mientras la progresiva crisis que atraviesa la Argentina produce que comportamientos similares se registren en comedores escolares del Gran Buenos Aires (Granovsky, 2016).

Entonces, parafraseando a Carli (2006) cuando sostiene que la infancia constituye un analizador privilegiado del tiempo presente, nos preguntamos: ¿qué tipo de subjetividad construye una política pública que observa como un problema que un niño comparta alimentos con sus familiares más cercanos? Por otro lado, ¿las políticas gubernamentales no dan los resultados esperados porque los niños comparten la comida? Estas cuestiones expresan las tensiones entre el modelo de infancia que sustentan las intervenciones sociales y los modos en que la infancia es experimentada en contextos de diversidad y desigualdad.

\section{3) El campo de disputas del cuidado infantil}

El cuidado y la infancia son categorías casi indisociables en el campo de la salud. Pero no todo tipo de cuidado es entendido como adecuado, mucho menos si lo que se está tratando de resolver son los déficits nutricionales. En la imagen universal de la infancia abonada por la pediatría, la madre emerge como el núcleo de todos los afectos y como la principal responsable del cuidado y la crianza de los más pequeños (Jenks, 1996; Colangelo, 2006). La "crianza correcta" implica una definición de las formas socialmente adecuadas de cuidar un niño, "preponderadamente en el ámbito privado, ligadas a la idea de normalidad, y por contraste, de aquellas que no lo son" (Colangelo, 2006, p.11). En los dos casos analizados, podemos observar cómo la acción estatal tiene por objetivo rehabilitar el cuidado inadecuado que reciben en sus casas. En el caso colombiano, se entiende que los niños deben acudir todos los días al hogar comunitario no solo porque necesiten acceder a la alimentación que no pueden obtener en sus casas, sino porque el cuidado que reciben allí no es del todo adecuado para el desarrollo de su "capital humano". En el caso argentino, la agente sanitaria en cuestión no solo se mostró preocupada por la emergencia de déficits nutricionales en su sector de salud, sino por la falta de rasgos civilizatorios de las familias wichís, cuyos niños "son como animalitos" y están "mal cuidados". 
La referencia a la animalidad merece atención especial, ya que remite a los modos en que se organizan las relaciones interétnicas en cada contexto. La alteridad juega un rol preponderante en el modo en que los agentes estatales ponen en tela de juicio la forma en que las comunidades indígenas cuidan a sus hijos. En el caso colombiano, el fuerte sentido de pertenencia y la autonomía de los niños puinave resulta un obstáculo para las intervenciones del ICBF, que suponen un niño más dependiente, que actúa a la espera del permiso de los adultos. La promoción de la autonomía infantil durante el proceso de socialización ha sido registrada en diversos contextos indígenas (Paradise, 1994; Szulc, 2015). En este sentido, Tassinari (2007) señala el reconocimiento de la autonomía de los niños y de su capacidad de decisión en diversas esferas de la vida social, incluyendo en ellas el acto alimentario, como así también lo advierte Szulc (2015) para el caso mapuche en Argentina. Asimismo, Alvares (2012) señala las tensiones en torno a la autonomía de los niños maxakali, que en sus comunidades deciden el momento de comer y luego son alimentados en la boca en los comedores estatales. En este campo de tensiones en torno a la infancia y su cuidado, las diferencias étnicas adquieren "significados arbitrarios que operan en base a supuestos implícitos acerca de atributos que se consideran innatamente generales", lo cual genera procesos de significación hegemónica que biologizan aspectos culturales (Briones, 1998, p. 7). En estas coordenadas de significados, emergen los "argumentos culturalistas" (Lorenzetti, 2015) por parte de funcionarios políticos, en los cuales "la cultura es comprendida como origen y causa a través de la cual se reproduce el padecimiento" (Leavy, 2015, p. 5): "Las muertes de niños en el norte de nuestra provincia son un problema cultural, no sanitario ni social, porque los aborígenes no concurren al hospital" (La política online, 9/2/2011) o "son nómades y no pueden ser atendidos" (Clarín, 7/2/2015). De este modo, los déficits nutricionales son entendidos como el resultado del cuidado inadecuado realizado por los adultos indígenas, sobre todo por sus madres. Asimismo, la diversidad cultural indígena es entendida como un conjunto de atributos que obstaculizan el acceso a los niños indígenas a pautas adecuadas de crianza (Colangelo, 2008).

\section{Reflexiones finales}

El análisis etnográfico de las intervenciones estatales sobre la infancia permite atender a las racionalizaciones, los supuestos y los esquemas interpretativos que orientan las prácticas concretas y las categorías que han sido construidas sobre ellas.

En primer lugar, nos parece relevante resaltar que las acciones focalizadas analizadas se fundan en una mirada descontextualizada de la infancia, que se encuentra íntimamente vinculada con la responsabilización de los propios sujetos promovida por la gubernamentalidad neoliberal. No negamos la necesidad de intervenir en la alimentación infantil durante los primeros años de vida, pero advertimos que dichas acciones no revertirán los procesos sociales, económicos y políticos que generan la inseguridad alimentaria (Osorio Figueroa y Bahia Coelho, 2017).

En segundo lugar, nos parece importante remarcar el carácter paradójico de las acciones estatales que se hacen en pos de la "seguridad alimentaria", siendo este un concepto que se elabora para entender el hambre no como un déficit de calorías sino como "una violación de un espectro más amplio de condiciones físicas, sociales y económicas" (Patel, 2012, p.3). No obstante, las acciones directas en territorio son diseñadas desde una perspectiva meramente nutricional y médica, que considera a los alimentos como nutrientes, vitaminas y/o minerales, sin atender a los condicionantes culturales e ideológicos que afectan los patrones de consumo (Hintze, 1989). Al respecto, Ortale (2000) señala que los alimentos cumplen funciones sociales, psicológicas y rituales y que, por lo tanto, no son objetos destinados únicamente a satisfacer necesidades biológicas. Advertimos entonces una continuidad entre la mirada descontextualizada de la 
alimentación, concebida únicamente en términos biológicos, y la noción de infancia, también descontextualizada, que subyace a dichas intervenciones.

En tercer lugar, desde la perspectiva antropológica, señalamos que el contexto en el cual se inscriben las intervenciones sanitarias no es una cuestión externa que debe ser relacionada con las experiencias subjetivas, ni meramente un escenario en el que las experiencias transcurren, sino justamente una parte constitutiva de ellas (Grimberg, 2009, p. 11). La emergencia de déficit nutricionales está en estrecha relación con la situación territorial que enfrentan los pueblos indígenas, signada por la deforestación, la tala indiscriminada, el avance de la frontera agropecuaria, la prospección petrolera, la expulsión de familias y comunidades hacia las ciudades, la contaminación de agua, la degradación de suelos (Buliubasich, 2013; Mampaey y Van Velde, 2015; FAO, 2015; Lorenzetti, 2015).

En cuarto lugar, se impone enfatizar la paradójica situación en que se encuentran los niños y niñas indígenas, en tanto titulares de derechos universales $y$, a la vez, de derechos específicos, que sin embargo, por medio de políticas como las analizadas quedan reducidos a derechos sociales básicos y medidas paliativas de la pobreza (Szulc et al., 2016). Esto involucra una persistente negación de la incidencia que la vulneración de los derechos colectivos de los pueblos indígenas tiene sobre las vidas de los niños, en consonancia con una marcada tendencia de la política indigenista local de reconocer a los pueblos indígenas como "sujetos de asistencia", en lugar de como "sujetos de derecho" (Lenton y Lorenzetti, 2005, p. 319).

En síntesis, consideramos que, así como la desnutrición no puede abordarse escindida de los aspectos estructurales y culturales involucrados en la alimentación, no podemos hablar de niños y niñas sin considerar el entramado de relaciones sociales del cual forman parte. Hablar del "sufrimiento de los niños", sin considerar las condiciones de vida de los adultos con quienes viven, elabora mecanismos perversos que estigmatizan a los que padecen y ocultan el eje de las responsabilidades (Buliubasich y Rodríguez, 2009). 


\section{Q Referencias bibliográficas}

" Alvares, M. (2012). Criança y comensalidade. A práticas alimentares y a construçao da persoa. En 54 Congreso Internacional de Americanistas. Viena, Austria.

" Álvarez Leguizamón, S. (2011). Gubernamentalidad neoliberal y focopolítica en América Latina: Los Programas de Transferencia Condicionadas. ¿Políticas de cohesión social? En C. Barba Solano (comp.), Perspectivas críticas sobre la cohesión social: Desigualdad y tentativas fallidas de integración social en América Latina (pp. 251-285). Buenos Aires: Consejo Latinoamericano de Ciencias Sociales.

"Andiñach, R. (2014). Impacto de la Asignación Universal por Hijo y el Programa Ciudadanía Porteña en el Consumo de Alimentos. (tesis de maestría). Facultad Latinoamericana de Ciencias Sociales, Buenos Aires, Argentina.

»Aulicino, C. (2012). Una primera aproximación a las políticas de Educación Nutricional en las provincias argentinas. Documento de Trabajo $N^{\circ}$ 90. Buenos Aires: Centro de Implementación de Políticas Públicas para la Equidad y el Crecimiento (CIPPEC). Recuperado de http://www.cippec.org/wp-content/uploads/2017/03/2412.pdf

» Aulicino, C. y Díaz Langou, G. (2012). La implementación del Plan Nacional de Seguridad Alimentaria en ámbitos subnacionales. Documento de Trabajo № 88. Buenos Aires: Centro de Implementación de Políticas Públicas para la Equidad y el Crecimiento (CIPPEC).

» Briones, C. (1998). La alteridad del “Cuarto Mundo”. Una deconstrucción antropológica de la diferencia. Buenos Aires: Ediciones del Sol.

» Buliubasich, C. (2013). La política indígena en Salta. Límites, Contexto etnopolítico y luchas recientes. Runa, 34(1), 59-71.

» Buliubasich, C. y Rodríguez, H. (2009). Panorama histórico y ambiental. En C. Buliubasich y A. González (Coords.), Los pueblos indígenas de Salta. La posesión y el dominio de sus tierras. Departamento de San Martín (pp. 25-35). Salta: Centro Promocional en Investigaciones en Historia y Antropología, Universidad de Salta.

"Calvo, E. y Aguirre, P. (2005). Crisis de la seguridad alimentaria en la Argentina y estado nutricional de una población vulnerable. Archivos Argentinos de Pediatría, 1(103), 77-90.

"Carli, S. (2006). La cuestión de la Infancia. Entre la escuela, la calle y el shopping. Buenos Aires: Paidós.

"Castillo Matamoros, S. E. (2009). La génesis del programa de hogares comunitarios del Instituto Colombiano de Bienestar Familiar. (tesis de doctorado). Centro de Estudios Avanzados en Niñez y Juventud, Universidad De Manizales, Manizales, Colombia. Recuperado de http://repository.cinde.org.co/handle/20.500.11907/527

"Comisión Económica para América Latina y el Caribe (CEPAL). 2007. Políticas y programas de salud de pueblos indígenas en América Latina. En Panorama Social de América Latina. Santiago: CEPAL. Recuperado de https://repositorio.cepal.org/bitstream/handle/11362/39965/S1600175_es.pdf

"Colangelo, M. A. (2006). La crianza en disputa. Un análisis del saber médico sobre el cuidado infantil. En VIII Congreso Argentino de Antropología Social. Salta, Argentina: Universidad Nacional de Salta

"Colangelo, M. A. (2008). La constitución de la niñez como objeto de estudio e intervención médicos en la Argentina de comienzos del siglo XX. En IX Congreso Argentino de Antropología Social. Posadas, Argentina: Universidad Nacional de Misiones. 
»Cravino, M. C. (2008). Los mil barrios (in)formales. Aportes para la construcción del hábitat popular del Área Metropolitana de Buenos Aires. Buenos Aires: Universidad Nacional de General Sarmiento.

»Daza Ramos, L. V. (2015). Integration of ICT into an adult education program for indigenous communities. The case of Guainía, Colombia. (tesis de maestría). Universitetet i Oslo, Oslo, Noruega. Recuperado de https://www.duo.uio.no/bitstream/handle/10852/49738/ Viviana-Daza-Ma--thesis.pdf?sequence $=1 \mathrm{el}$.

» Fassin, D. (2003). Gobernar por los cuerpos, políticas de reconocimiento hacia los pobres y migrantes en Europa. Cuadernos de Antropología Social, 17, 49-78.

»Food and Agriculture Administration (FAO). (2015). Los pueblos Indígenas y las Políticas de Seguridad Alimentaria Nutricional en América Latina y el Caribe. Una visión Regional. Resumen Ejecutivo. Recuperado de http://www.fao.org/3/a-i4828s.pdf

» Gordillo, G. (2006). Reciprocidad y diferenciación social. En En el Gran Chaco. Antropologías e historias (pp.125-146). Buenos Aires: Prometeo.

" Grimberg, M. (2009). Experiencias y narrativas de padecimientos cotidianos. En Miradas antropológicas sobre la salud, la enfermedad y el dolor crónico (pp. 21-52). Buenos Aires: Antropofagia.

» Guber, R. (2001). La etnografía: método, campo y reflexividad. Buenos Aires: Norma.

» Herkovits, D. (2008). La construcción de la malnutrición infantil: una etnografía sobre las condiciones y posibilidades que contribuyen a su producción y reproducción en hogares pobres de la Ciudad de Buenos Aires. (tesis de maestría). Centro de Estudios de Estado y Sociedad, Facultad Latinoamericana de Ciencias Sociales, Buenos Aires, Argentina.

» Hintze, S. (1989). Estrategias alimentarias de sobrevivencia. Un estudio de caso en el Gran Buenos Aires. Buenos Aires: Centro Editor de América Latina.

» Instituto Nacional de Estadística y Censos de la República Argentina (INDEC) (2014). Encuesta Permanente de Hogares. Recuperado de http://www.indec.gov.ar/informesdeprensa_anteriores.asp?id_tema_1=4\&id_tema_2=31\&id_tema_3=58.

» Ingold, T. (1988). Notes on the Foraging Mode of Production. En T. Ingold, D. Riches, y J. Woodburn (Eds.), Hunters and Gatherers 1. History, Evolution and Social Change (pp. 269285). Nueva York: St. Martin's Press.

» Jenks, C. (1996). Childhood. Londres: Routledge.

» Leavy, M. P. (2014). Aportes del enfoque antropológico para el estudio de infestaciones parasitarias. En S. Hirsch, D. Salomón y M. Lorenzetti (Comps.), Procesos de Investigación e Intervención en Salud en Comunidades Indígenas de la Argentina (pp. 109-151). Iguazú: Instituto Nacional de Medicina Tropical del Ministerio de Salud de la Nación.

» Leavy, M. P. (2015). Aportes para pensar el flagelo de la desnutrición desde la antropología de la infancia. Horizontes Sociológicos, 3(6), 54-72. Recuperado de: http://aass.org. elserver.com/ojs/index.php/hs/issue/view/5/showToc

» Lee, R. (1981). Is there a Foraging Mode of Production? Canadian Journal of Anthropology, 2, 13-19.

» Lenton, D. y Lorenzetti, M. (2005). Neoindigenismo de necesidad y urgencia: la inclusión de los Pueblos indígenas en la agenda del estado neoasistencialista. En C. Briones (Ed.), Cartografías Argentinas. Políticas indigenistas y formaciones provinciales de alteridad (pp. 293-325). Buenos Aires: Antropofagia.

» Llobet, V. (2011). Las políticas para la infancia y el enfoque de derechos en América Latina. Algunas reflexiones sobre su abordaje teórico. Fractal: Revista de Psicología, 23(3), 447-460. 
»Lorenzetti, M. (2007). Del "capital social" a la "culturalización de la pobreza": la construcción de alteridad en los programas de salud para la población indígena. En 4tas Jornadas de Jóvenes Investigadores. Instituto de Investigaciones Gino Germani, Facultad de Ciencias Sociales, Universidad de Buenos Aires, Buenos Aires, Argentina.

» Lorenzetti, M. (2015). “Luchas de acento”: Salud-Enfermedad-Atención en las comunidades wichís de Tartagal (Salta, Argentina). En S. Hirsch, D. Salomón y M. Lorenzetti (Comps.), Procesos de investigación e intervención en comunidades indígenas de la Argentina (pp. 71-109). Misiones: Instituto Nacional de Medicina Tropical del Ministerio de Salud de la Nación.

» Maceira, D. (2010). Cómo fortalecer las redes de servicio de salud. El caso Salta. Documento de Políticas Públicas. Centro de Políticas Públicas para la Equidad y el Crecimiento. Recuperado de http://www.cippec.org/-/como-fortalecer-las-redes-de-servicios-desalud-el-caso-de-salta.

» Mampaey, M. y Van Velde, E. (2015). Retardo de crecimiento o “stunted growth". Prevalencia y repercusión en la salud de niños Mbya Guaraní de las comunidades de Ruiz de Montoya, Misiones. En S. Hirsch, D. Salomón y M. Lorenzetti (Comps.), Procesos de Investigación e intervención en comunidades indígenas de la Argentina (pp. 245-261). Misiones: Instituto Nacional de Medicina Tropical del Ministerio de Salud de la Nación.

» O’Donnell, A. y Carmuega, E. (1998). La transición epidemiológica y la situación nutricional de nuestros niños. Boletín del Centro de Estudios sobre Nutrición Infantil (CESNI). Recuperado de http://biblioteca.cesni.org.ar/references/2dacd804a73d499417120000

» Organización de las Naciones Unidas (ONU). (2016). Declaración de las Naciones Unidas sobre los Derechos Pueblos Indígenas. Recuperado de http://www.un.org/es/events/indigenousday/pdf/indigenousdeclaration_faqs.pdf.

» Ortale, S. (2000). Saber médico y desnutrición en el Gran La Plata. Latin American Studies Association. Miami, USA. Recuperado de http://lasa.international.pitt.edu/Lasa20oo/Ortale.PDF

» Osorio Figueroa, C. D. y Bahia Coelho, T. C. (2017). Infancia maya guatemalteca: vulnerabilidad nutricional y políticas públicas para su enfrentamiento. DESIDADES-Revista Eletrônica de Divulgação Científica da Infância e Juventude, 15. Recuperado de http://desidades.ufrj.br/es/featured_topic/infancia-maya-guatemalteca-vulnerabilidad-nutricional-y-politicas-publicas-para-su-enfrentamiento/.

»Paradise, R. (1994). La socialización para la autonomía en un contexto interaccional mazahua. En L. E. Galván, M. Lamoneda, M. E. Vargas y S. Calvo (Coords.). Memorias del Primer Simposio de Educación (pp. 483-490). México: Centro de Investigación y Estudios Superiores en Antropología Social (CIESAS).

»Patel, R. (2012). Soberanía alimentaria: poder, género y el derecho a la alimentación. Public Library of Science Medicine, 9(6), 1-4.

» Rose, N., O`Malley, P. y Valverde, M. (2006). Governmentality. Annual Review Law Society Science 2, 83-104. Recuperado de http://www.annualreviews.org/doi/pdf/10.1146/annurev.lawsocsci.2.081805.105900

»Segato, R. (2013). Que cada pueblo teja los hilos de su historia: la colonialidad legislativa de los salvadores de la infancia indígena. En La crítica a la colonialidad en ocho ensayos (pp. 113-153). Buenos Aires: Prometeo.

»Szulc, A. (2006). Antropología y niñez: de la omisión a las ‘culturas infantiles’. En G. Wilde y P. Schamber (Eds.), Cultura, comunidades y procesos contemporáneos (pp. 25-51). Buenos Aires: SB. 
»Szulc, A. (2015). La niñez mapuche en el Neuquén. Sentidos de pertenencia en tensión. Buenos Aires: Biblos.

» Szulc, A., Colangelo, M. A., Shabel, P., Leavy, M. P., Enriz, N., y Hernández, M. C. (2016). $\mathrm{Al}$ rescate de la niñez indígena. Reflexiones antropológicas a partir de una campaña de UNICEF Argentina. Política y Sociedad, 53(1), 123-142.

» Tassinari, A. (2007). Concepções Indígenas de Infância no Brasil. 53 Congreso Internacional de Americanistas. Escuela Nacional de Antropología e Historia, Ciudad de México, México.

» Teubal, M. (2006). Transformaciones en el Sistema Agroalimentario y acceso a la Alimentación. Revista Realidad Económica, 196. Recuperado de http://www.iade.org.ar/noticias/soja-transgenica-y-crisis-del-modelo-agroalimentario-argentino.

» Torres Aliaga, T. y Torres Secchi, A. (2010). ¿Por qué callan si nacen llorando? Poder, accesibilidad y diferencias culturales en Salud. Iruya, 1978-2008. Formosa: Endepa.

»Valles, M. (2000). Técnicas cualitativas de investigación social. Reflexión metodológica y práctica profesional. Madrid: Síntesis.

\section{Otras fuentes consultadas}

»Aranda, D. (2014). Deforestación. Salta es la líder de provincias del país. Revista Norte, 30 de septiembre de 2014. Recuperado de http://revistanorte.com.ar/deforestacion-saltaes-lider-entre-las-provincias-del-pais/.

»Clarín Diario (7 de febrero de 2015). Urtubey: la desnutrición infantil en Salta existe y todavía nos sigue golpeando. Sociedad. Recuperado de http://www.clarin.com/sociedad/ desnutricion_Urtubey_Salta_0_1299470102.html el 13/02/2015 (15 de febrero de 2016).

»El Tiempo, 14 de marzo de 2016. Lo que no comemos: Guainía. Hambre en Guainía. Casa Editorial. Recuperado de http://www.eltiempo.com/multimedia/especiales/escasez-dealimentos-y-desnutricion-en-colombia/16536975/1/ (2 de agosto de 2017).

»El Tribuno, 21 de abril de 2015. La situación de las familias de Salta está en las peores del país. Sociedad. Recuperado de http://www.eltribuno.info/la-situacion-economica-lasfamilias-saltenas-esta-las-peores-del-pais-n537684 (7 de agosto de 2017)

» Granovsky, Martín. Crónica del chico que se guarda el flancito. Página 12, 17 de julio de 2016. Recuperado de http://www.pagina12.com.ar/diario/sociedad/3-304478-2016-07-17. html (18 de agosto de 2016).

» Instituto Colombiano de Bienestar Familiar-ICBF (2014) Lineamientos Técnico Administrativo y Operativo Hogares comunitarios en todas sus formas (FAMI, Familiares, Grupales, Múltiples, Múltiples Empresariales y Jardines Sociales) para la atención a los niños y niñas hasta los cinco(5) años de edad. Recuperado de https://www.icbf.gov.co/sites/default/files/anexo_5._lineamiento_hogares_comunitarios_bnopi_definitivo_octubre.pdf.

" La política Online, 9 de febrero de 2011. Según Urtubey, los aborígenes desnutridos no van al hospital "por una cuestión cultural". Sociedad. Recuperado de http://www.lapoliticaonline.com/nota/50049/.

»Presidencia de La República de Colombia (2013). De Cero a Siempre. Estrategia de atención integral a la primera infancia: fundamentos políticos, técnicos y de gestión. Recuperado de: http://www.deceroasiempre.gov.co/Prensa/CDocumentacionDocs/ Fundamientos-politicos-tecnicos-gestion-de-cero-a-siempre.pdf. 